\title{
Oscillation of Second-Order Nonlinear Delay Dynamic Equations on Time Scales
}

\author{
X. Song, Q.X. Zhang \\ Department of Mathematics \\ Binzhou University \\ Shandong, China
}

\begin{abstract}
By using the generalized Riccati transformation and the inequality technique, we established one new oscillation criterion for the second-order nonlinear delay dynamic equations on time scales. Our results not only extend and improve some known theorems, but also unify the oscillation of the second-order nonlinear delay differential equation and the second-order nonlinear delay difference equation on time scales.
\end{abstract}

Keywords-oscillation criterion; dynamic equations; time scale

\section{INTRODUCTION}

The theory of time scales, which has recently received a lot of attention, was introduced by Hilger [1], in order to unify continuous and discrete analysis. Several authors have expounded on various aspects of this new theory, see [2]. A time scale $\mathrm{T}$ is an arbitrary closed subset of the reals and the cases when this time scale is equal to the reals or to the integers represent the classical theories of differential and of difference equations. In recent years, there has been much research activity concerning the oscillation and nonoscillation of solutions of various equations on time scales, and we refer the reader to [3-10]. In this paper we deal with the oscillatory behavior of all solutions of nonlinear second-order delay dynamic equation

$$
\left.\left(a(t) x^{\Delta}(t)\right)^{\gamma}\right)^{\Delta}+q(t) f(x(\tau(t)))=0, \quad t \in \mathrm{T}, \quad t \geq t_{0} .
$$

In this paper, we give the following hypotheses:

(H1) $\mathrm{T}$ is a time scale (i.e., a nonempty closed subset of the real numbers $\mathrm{R}$ ) which is unbounded above, and $t_{0} \in \mathrm{T}$ with $t_{0}>0$. We define the time scale interval of the form $\left[t_{0}, \infty\right)_{\mathrm{T}}$ by $\left[t_{0}, \infty\right)_{\mathrm{T}}=\left[t_{0}, \infty\right) \cap \mathrm{T}$.

(H2) $\gamma \geq 1$ is the ratio of two positive odd integers.

(H3) $a, q:$ are positive real-valued rd-continuous functions on an arbitrary time scale $\mathrm{T}$, and

$$
\int_{t_{0}}^{\infty}\left(\frac{1}{a(t)}\right)^{\frac{1}{\gamma}} \Delta t<\infty \text {. }
$$

(H4) $\tau: \mathrm{T} \rightarrow \mathrm{T}$ is a strictly increasing and differentiable function such that

$$
\tau(t) \leq t, \lim _{t \rightarrow \infty} \tau(t)=\infty \text {, and } \tau(\mathrm{T}) \subset \mathrm{T} \text {. }
$$

(H5) $f: \mathrm{R} \rightarrow \mathrm{R}$ is a continuous function such that satisfies for some positive constant $L$,

$$
\frac{f(x)}{x^{r}} \geq L \text { for all } x \neq 0 .
$$

This article is the continuation of [9]. In this paper, suppose that the condition

$$
\int_{t_{0}}^{\infty}\left(\frac{1}{a(t)}\right)^{\frac{1}{\gamma}} \Delta t=\infty
$$

is invalid, we continue to discuss the oscillation of solutions of (1). By using the generalized Riccati transformation and the inequality technique, we obtain one new oscillation criteria for (1).

By a solution of (1), we mean a nontrivial real-valued function $x$ satisfying (1) for $t \in \mathrm{T}$. We recall that a solution $x$ of (1) is said to be oscillatory on $\left[t_{0}, \infty\right)_{\mathrm{T}}$ in case it is neither eventually positive nor eventually negative; otherwise, the solution is said to be nonoscillatory. Equation (1) is said to be oscillatory in case all of its solutions are oscillatory. Our attention is restricted on those solutions $X$ of (1) which $X$ is not the eventually identically zero.

On the time scale $\mathrm{T}$ we define the forward and the backward jump operators by

$$
\sigma(t)=\inf \{s \in T: s>t\} \text { and } \rho(t)=\sup \{s \in T: s<t\} .
$$

A point $t \in \mathrm{T}$ is said to be left-dense if $\rho(t)=t$, rightdense if $\sigma(t)=t$, left-scattered if $\rho(t)<t$ and right-scattered if $\sigma(t)>t$. The graininess $\mu$ of the time scale is defined by $\mu(t)=\sigma(t)-t$. For a function $f: \mathrm{T} \rightarrow \mathrm{R}$, if $f$ is continuous at $t$ and $t$ is right-scattered, the (delta) derivative is defined by

$$
f^{\Delta}(t)=\frac{f(\sigma(t))-f(t)}{\sigma(t)-t} .
$$

If $t$ is right-dense, then the derivative is defined by 


$$
f^{\Delta}(t)=\lim _{s \rightarrow t^{+}} \frac{f(t)-f(s)}{t-s}
$$

provided this limit exists. We will make use of the following product and quotient rules for the derivative of the product $f g$ and the quotient $f / g$ of two differentiable functions $f$ and $g$ :

$$
(f g)^{\Delta}(t)=f^{\Delta}(t) g(t)+f(\sigma(t)) g^{\Delta}(t)=f(t) g^{\Delta}(t)+f^{\Delta}(t) g(\sigma(t)),
$$

$$
\left(\frac{f}{g}\right)^{\Delta}(t)=\frac{f^{\Delta}(t) g(t)-f(t) g^{\Delta}(t)}{g(t) g(\sigma(t))} \quad \text { if } \quad g \cdot g^{\sigma} \neq 0 .
$$

For $b, c \in \mathrm{T}$ and a differentiable function $f$, the Cauchy integral of $f^{\Delta}$ is defined by

$$
\int_{b}^{c} f^{\Delta}(t) \Delta t=f(c)-f(b)
$$

The integration by parts formula reads

$$
\int_{b}^{c} f^{\Delta}(t) g(t) \Delta t=f(c) g(c)-f(b) g(b)-\int_{b}^{c} f^{\sigma}(t) g^{\Delta}(t) \Delta t
$$

For more details, see [2].

\section{SEVERAL LEMMAS}

Lemma 1 [10, Lemma 2.2] Assume that $\tau: \mathrm{T} \rightarrow \mathrm{R}$ is strictly increasing and $\tau(\mathrm{T}) \subset \mathrm{T}$ is a time scale and $\tau(\sigma(\mathrm{t}))=\sigma(\tau(\mathrm{t}))$. Let $x: \mathrm{T} \rightarrow \mathrm{R}$. If $\tau^{\Delta}(\mathrm{t})$, and let $x^{\Delta}(\tau(t))$ exist for $t \in \mathrm{T}$, then $(x(\tau(t)))^{\Delta}$ exist, and

$$
(x(\tau(t)))^{\Delta}=x^{\Delta}(\tau(t)) \tau^{\Delta}(t) .
$$

Lemma 2 [2, Theorem 1.90] Assume that $x(t)$ is deltadifferentiable and eventually positive or eventually negative, then

$$
\left((x(t))^{\gamma}\right)^{\Lambda}=\gamma \int_{0}^{1}[h x(\sigma(t))+(1-h) x(\mathrm{t})]^{\gamma-1} x^{\Lambda}(t) d h .
$$

Lemma 3[11, Theorem 41] Assume that $X$ and $Y$ are nonnegative real numbers, then

$$
\lambda X Y^{\lambda-1}-X^{\lambda} \leq(\lambda-1) Y^{\lambda}, \quad \lambda>1,
$$

where the equality holds if and only if $X=Y$.

\section{MAIN RESUlts}

Theorem 1 Assume (H1)-(H5), (2) hold and $\tau \in C_{r d}^{1}\left(\left[t_{0}, \infty\right)_{\mathrm{T}}, \mathrm{T}\right), \tau(\sigma(t))=$

$$
\sigma(\tau(t)) \text {. Let } H: D_{\mathrm{T}}=\left\{(t, s): t \geq s \geq t_{0}, t, s \in\left[t_{0}, \infty\right)_{\mathrm{T}}\right\} \rightarrow \mathrm{R} \text { be }
$$
a rd-continuous function, which is such that

$$
H(t, t)=0 \text { for } t \geq t_{0}, H(t, s)>0 \text { for } t>s \geq t_{0}, t, s \in\left[t_{0}, \infty\right)_{\mathrm{T}} .
$$

And $H$ has a non-positive continuous $\Delta$-partial derivative $H^{\Delta s}(t, s)$ with respect to the second variable, if there exists a positive $\Delta$-differentiable function $\delta \in C_{r d}^{1}\left(\left[t_{0}, \infty\right)_{T}, \mathrm{R}\right)$, such that

$$
\lim _{t \rightarrow \infty} \sup \frac{1}{H\left(t, t_{0}\right)} \int_{t_{0}}^{f} H(t, s)\left[L \delta(s) \mathrm{q}(s)-\frac{a(\tau(s))\left(\delta^{\Delta}(s)\right)_{+}^{\gamma+1}}{(\gamma+1)^{\gamma+1}\left(\delta(s) \tau^{\star}(s)\right)^{\gamma}}\right] \Delta s=\infty,
$$
$t_{1} \in\left[t_{0}, \infty\right)_{\mathrm{T}}$

where $\left(\delta^{\Delta}(t)\right)_{+}=\max \left\{0, \delta^{\Delta}(t)\right\}, t \in\left[t_{1}, \infty\right)_{\mathrm{T}}$. If for every

$$
\int_{t_{1}}^{\infty}\left[\frac{1}{a(s)} \int_{t_{1}}^{s} \theta^{y}(u) q(u) \Delta u\right]^{\frac{1}{\gamma}} \Delta s=\infty,
$$

where $\theta(t)=\int_{t}^{\infty}\left(\frac{1}{a(s)}\right)^{\frac{1}{y}} \Delta s$, then equation (1) is oscillatory on $\left[t_{0}, \infty\right)_{\mathrm{T}}$.

Proof Suppose to the contrary that $x(t)$ is a nonoscillatory solutions of equation (1) on $\left[t_{0}, \infty\right)_{\mathrm{T}^{*}}$ we may assume without loss of generality that $x(t)>0$ and $x(\tau(t))>0$ for all $t \in\left[t_{0}, \infty\right)_{\mathrm{T}}, t_{1} \in\left[t_{0}, \infty\right)_{\mathrm{T}}$ We shall consider only this case, since the proof when $X$ is eventually negative is similar. By equation (1), we have $\left(a(t)\left(x^{\Delta}(t)\right)^{\gamma}\right)^{\Delta}<0$. Since $a(t)\left(x^{\Delta}(t)\right)^{\gamma}$ is decreasing, it is eventually of one sign and hence ${ }^{x^{\Delta}(t)}$ is eventually of one sign. Thus, we shall distinguish the following two cases:

$$
\begin{aligned}
& \text { (I) } x^{\Delta}(t)>0 \text { for } t \geq t_{1} \text {; and } \\
& \text { (II) } x^{\Delta}(t)<0 \text { for } t \geq t_{1}
\end{aligned}
$$

Case (I) The proof that ${ }^{x^{\Delta}(t)}$ is an eventually positive is similar to that of the proof of Theorem 4.1 in [9] and it hence is omitted.

Case (II) For $s \geq t \geq t_{1}$, we have

$$
a(s)\left(-x^{\Delta}(s)\right)^{\gamma} \geq a(t)\left(-x^{\Delta}(t)\right)^{\gamma}
$$

and hence

$$
-x^{\Delta}(s) \geq\left(\frac{a(t)}{a(s)}\right)^{\frac{1}{y}}\left(-x^{\Delta}(t)\right)
$$

Integrating (11) from $t \geq t_{1}$ to $u \geq t$ and letting $u \rightarrow \infty$ yields

$$
\begin{aligned}
& x(t) \geq\left[\int_{t}^{\infty}\left(\frac{1}{a(s)}\right)^{\frac{1}{y}} \Delta s\right](a(t))^{\frac{1}{y}}\left(-x^{\Delta}(t)\right)=-\theta(t) x^{\Delta}(t) \\
& \text { for } t \in\left[t_{1}, \infty\right)_{\mathrm{T}} \text {, and thus } \\
& (x(t))^{r} \geq-(\theta(t))^{\gamma} a(t)\left(x^{\Delta}(t)\right)^{\gamma} \\
& \geq-(\theta(t))^{\gamma} a\left(t_{1}\right)\left(x^{\Delta}\left(t_{1}\right)\right)^{\gamma}=b(\theta(t))^{\gamma}
\end{aligned}
$$


we find

$$
\begin{gathered}
-\left(a(t)\left(x^{\Delta}(t)\right)^{\gamma}\right)^{\Delta} \geq L q(t)(x(\tau(t)))^{\gamma} \\
\geq L q(t)(x(t))^{\gamma}=b L(\theta(t))^{\gamma} q(t)
\end{gathered}
$$

for $t \in\left[t_{1}, \infty\right)_{\mathrm{T}}$. Integrating (13) from $t_{1}$ to $t$ we have

$$
\begin{aligned}
-a(t)\left(x^{\Delta}(t)\right)^{\gamma} & \geq-a\left(t_{1}\right)\left(x^{\Delta}\left(t_{1}\right)\right)^{\gamma}+b L \int_{t_{1}}^{t}(\theta(s))^{\gamma} q(s) \Delta s \\
& \geq b L \int^{t}(\theta(s))^{\gamma} q(s) \Delta s
\end{aligned}
$$

such that

$$
-x^{\Delta}(t) \geq\left[\frac{b L}{a(t)} \int_{h_{1}}^{t}(\theta(s))^{r} q(s) \Delta s\right]^{\frac{1}{\gamma}} .
$$

Integrating (14) from ${ }^{t_{1}}$ to $t$, we obtain

$$
\infty>x\left(t_{1}\right) \geq-x(t)+x\left(t_{1}\right) \geq \int_{4}^{t}\left[\frac{b L}{a(s)} \int_{4}^{s} \theta^{\prime}(u) q(u) \Delta u\right]^{\frac{1}{4}} \Delta s \rightarrow \infty
$$

as $t \rightarrow \infty$. By (10), we get a contradiction. This completes the proof.

Remark 2 From Theorem1, we can obtain different conditions for oscillation of all solutions of (1) with different choices of $\delta(t)$ and $H(t, s)$. For example, $H(t, s)=(t-s)^{m}$ or $H(t, s)=\left(\ln \frac{t+1}{s+1}\right)^{m}$.

Now, let us consider the function ${ }^{H(t, s)}$ defined by

$$
H(t, s)=(t-s)^{m}, \quad m \geq 1, t \geq s \geq t_{0}, t, s \in\left[t_{0}, \infty\right)_{\mathrm{T}},
$$

Then $H(t, t)=0$ for $t \geq t_{0}$, and $H(t, s)>0, H^{\Delta}(t, s) \leq 0$, for $t>s \geq t_{0}, t, s \in\left[t_{0}, \infty\right)_{\mathrm{T}}$. Hence we have the following results.

Corollary 3 Assume (H1)-(H5), (2) , (10) hold and $\tau \in C_{r d}^{1}\left(\left[t_{0}, \infty\right)_{\mathrm{T}}, \mathrm{T}\right), \tau(\sigma(t))=\sigma(\tau(t))$. If there exists a positive $\Delta$-differentiable function $\delta \in C_{r d}^{1}\left(\left[t_{0}, \infty\right)_{\mathrm{T}}, \mathrm{R}\right)$ and $m \geq 1$ such that

$$
\operatorname{limssp}_{t \rightarrow \infty} \frac{1}{t^{m}} \int_{t_{0}}^{t}(t-s)^{m}\left[L \delta(s) \mathrm{q}(s)-\frac{a(\tau(s))\left(\delta^{\Delta}(s)\right\}_{+}^{\gamma+1}}{(\gamma+1)^{\gamma+1}\left(\delta(s) \tau^{\Delta}(s)\right)^{\gamma}}\right] \Delta \mathrm{s}=\infty,
$$

for $t>s \geq t_{0}, t, s \in\left[t_{0}, \infty\right)_{\mathrm{T}}$, then equation (1) is oscillatory on $\left[t_{0}, \infty\right)_{\mathrm{T}}$.

Remark 4 In the past, the usual result is that the conditions (2) was established, then every solution of the equation (1) is either oscillatory or converges to zero. But now Theorems 1 in our paper proved that if the condition (2) is satisfied, every solution of the equation (1) is oscillatory. Theorem 1 in this paper are new even in the cases $T==R$ and $\mathrm{T}:=Z$.

Example 5 Consider the second-order nonlinear delay 2difference equation

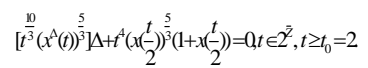

Here

$$
a(t)=t^{\frac{10}{3}}, q(t)=t^{4}, f(x)=x^{\frac{5}{3}}\left(1+x^{2}\right), \tau(t)=\frac{t}{2}, \gamma=\frac{5}{3} .
$$

The conditions (H1)-(H4) are clearly satisfied, (H5) holds with $L=1$. Next, for $t \geq 2$ so that

$$
\int_{i_{0}}^{\infty}\left(\frac{1}{a(t)}\right)^{\frac{1}{y}} \Delta t=\int_{2}^{\infty}\left(t^{\frac{10}{3}}\right)^{\frac{3}{5}} \Delta t=\int_{2}^{\infty} t^{-2} \Delta t=1<\infty \text { as } t \rightarrow \infty .
$$

Hence (2) is satisfied. Now let ${ }^{H(t, s)}=(t-s)^{2}, \delta(t)=t$, for all $t>s \geq 2$, then

$$
\begin{aligned}
& \lim _{t \rightarrow \infty} \sup \frac{1}{H\left(t, t_{0}\right)} \int_{t_{0}}^{t} H(t, s)\left[L \delta(s) q(s)-\frac{a(\tau(s))\left(\delta^{\Lambda}(s) \gamma_{\psi^{\gamma+1}}\right.}{(\gamma+1)^{\gamma+1}\left(\delta(s) \tau^{\Delta}(s)\right)^{\gamma}}\right] \Delta s \\
& =\lim _{t \rightarrow \infty} \sup \frac{1}{(t-2)^{2}} \int_{2}^{1}\left(s^{7}-2 t s^{6}+t^{2} s^{5}-b s^{\frac{11}{3}}+2 b t s^{\frac{8}{5}}-b t^{\frac{2}{5}} s^{\frac{5}{3}}\right) \Delta s=\infty,
\end{aligned}
$$

where $^{b=\left(\frac{1}{2}\right)^{\frac{5}{3}}\left(\frac{8}{3}\right)^{-\frac{8}{3}}}$, hence (9) is satisfied. Because of

$$
\theta(t)=\int_{i}^{\infty}\left(\frac{1}{a(s)}\right)^{\frac{1}{y}} \Delta s=\int_{i}^{\infty} s^{-2} \Delta s=-\frac{2}{s} t_{t}^{\infty}=\frac{2}{t},
$$

and thus

$$
\int_{4}^{0} \theta(u) q(u) \Delta u=\int_{2}^{2} \frac{2}{u} u^{4} \Delta u=\frac{2}{15}\left(s^{4}-s^{2}\right)=\frac{2}{15}\left(s^{2}+2^{2}\right)\left(s^{2}-2^{2}\right) \geq \frac{2}{15} s^{2},
$$

then

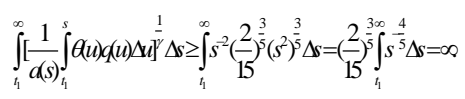

So (10) is satisfied as well. Altogether, by Theorem1, the equation (16) is oscillatory.

\section{ACKNOWLEDGEMENT}

This work was supported by grants from the Natural Science Foundation of Shandong Province of China (No. ZR2013AM003) and the Science Foundation of Young Talents of Binzhou University (No. BZXYQNLG201009).

\section{REFERENCES}

[1] S. Hiller, Analysis on measure chains-a unified approach to continuous and discrete calculus. Results Math., 1990, 18: 18-56.

[2] M. Bohner and A. Peterson, Dynamic Equations on Time Scales: An Introduction with Applications. Boston, Birkhauser, 2001.

[3] L. Erbe, Oscillation criteria for second order linear equations on a time scale. Can. Appl. Math. Q., 2001, 9: 345-375.

[4] L. Erbe, A. Peterson and P. Reh'ak, Comparison theorems for linear dynamic equations on time scales. J. Math. Anal. Appl., 2002, 275: 418-438.

[5] R. P. Agarwal, M. Bohner, D. O’Regan and A. Peterson, Dynamic equations on time scales: a survey. J. Comput. Appl. Math., 2002, 141: 1-26.

[6] R. P. Agarwal, M. Bohner and S. H. Saker, Oscillation of second order delay dynamic equations. Can. Appl. Math. Q., 2005, 13: 1-18.

[7] S. R. Grace, M. Bohner and R. P. Agarwal, On the oscillation of second-order half-linear dynamic equations. J. Difference Equ. Appl., 2009, 15: 451-460.

[8] L. Erbe, T. S. Hassan and A. Peterson, Oscillation criteria for nonlinear functional neutral dynamic equations on time scales. J. Difference Equ. Appl., 2009, 15: 1097-1116. 
[9] Q. Zhang, L. Gao and L. Wang, Oscillation of second-order nonlinear delay dynamic equations on time scales. Comput. Math. Appl., 2011, 61: 2342-2348.

[10] Z. Han, T. Li, S. Sun and C. Zhang, Oscillation for second-order nonlinear delay dynamic equations on time scales. Adv. Diff. Equ., 2009, 2009, Article ID 756171, 13pages.

[11] G. H. Hardy, J. E. Littlewood and G. Polya, Inequalities, Second Edition. Cambridge: Cambridge Univ. Press, 1988. 